\title{
A Survey on various image processing techinques for glaucoma diagnosis
}

\author{
Mrs. Kinjan Chauhan ${ }^{1}$, Dr. Ravi Gulati ${ }^{2}$ \\ ${ }^{1}$ Shree Ramkrishna Institute of Computer Education and Applied Sciences, Surat, Gujarat, India. \\ kinjanchauhan99@gmail.com \\ ${ }^{2}$ Department of Computer Science,Veer Narmad South Gujarat University,Surat, Gujarat, India.
} rmgulati@gmail.com

\begin{abstract}
Glaucoma is considered to be the leading cause for blindness in patients. Intra-Ocular Pressure(IOP) is one of the primary factors which plays an important role in the development of Glaucoma. There is a need for checking progression of Glaucoma in patients at early stage so that it's progression can be controlled and/or necessary action can be taken. In this paper, we have surveyed various image processing techniques used for detection of Glaucoma in patients using various opthalmic tests.
\end{abstract}

\section{Indexing terms/Keywords}

Glaucoma, OCT image, Perimetry, Image processing techniques

\section{Academic Discipline And Sub-Disciplines}

Computer Science

\section{SUBJECT CLASSIFICATION}

Image Processing

\section{Council for Innovative Research}

Peer Review Research Publishing System

Journal: International Journal of Management \& Information Technology

Vol. 5, No. 1

editor@cirworld.com

www.cirworld.com, member.cirworld.com 


\section{INTRODUCTION}

Glaucoma is the second leading cause of blindness after cataracts and its detection is essential to prevent visual damage. Glaucoma being the cause of blindness is 12.8 per cent of the total blindness in the country. In India, more than 90 per cent of Glaucoma in the community goes undiagnosed. In India, it is estimated that Glaucoma affects 12 million people and by 2020, this is expected to be 16 million. Statistics say that one in eight persons above the age of 40 years in India is either suffering from Glaucoma or is at the risk of the disease [1].

Diagnosis of Glaucoma depends on various findings such as elevated Intra Ocular Pressure (IOP $>23 \mathrm{~mm} \mathrm{Hg}$ is considered as a suspicious case for glaucoma), optical nerve cupping and visual field loss. Detection of Glaucoma is carried out by various tests such as Tonometry, Ophthalmoscopy, Perimetry, OCT, Gionoscopy, Pachymetry.[2]

\section{VARIOUS TECHINQUES FOR DETECTION OF GLAUCOMA}

Glaucoma diagnosis is a greatly researched topic. Various Methods such as Image processing, Artificial Neural Network, Neuro-Fuzzy etc have been applied for successful diagnosis of Glaucoma. Images obtained from OCT and fundus have been used widely for developing diagnosis [3][4].

Glaucoma is an optic neuropathy which is characterized by a gradual loss of retinal ganglion cells and thinning of the retinal nerve fibre layer. The functional assessment of glaucoma progression is determined by visual field testing. Glaucomatous Visual Field abnormality is detectable only after significant Retinal Nerve Fibre Layer (RNFL) loss has already occurred. In addition, Visual Field testing is prone to short- and long-term fluctuations as it is patient dependent and thus multiple testing is required to confirm any abnormalities. Assessment of the Optic Nerve Head (ONH) and the peripapillary RNFL can provide earlier indications of glaucomatous damage. However, the wide range of variation and the subjective nature of examination limit their reliability.

Glaucoma detection includes five tests

1. Tonometry

2. Ophthalmoscopy

3. Perimetry

4. Gonioscopy

5. Pachymetry

Tonometry measures the pressure within your eye. A small amount of pressure is applied to the eye by a tiny device or by a warm puff of air. The range for normal pressure is $12-22 \mathrm{~mm} \mathrm{Hg}$. Most glaucoma cases are diagnosed with pressure exceeding $20 \mathrm{~mm} \mathrm{Hg}$. However, some people can have glaucoma at pressures between $12-22 \mathrm{~mm} \mathrm{Hg}$. Eye pressure is unique to each person.

Ophthalmoscopy This diagnostic procedure helps in examining your optic nerve for glaucoma damage. Pupils of eye are to dilated so that the shape and color of the optic nerve can be examined.The opthalmologist doctor will then use a small device with a light on the end to light and magnify the optic nerve. If intraocular pressure is not within the normal range or if the optic nerve looks unusual, further perimetry and gonioscopy test are carried out.

Perimetry is a visual field test that produces a map of complete field of vision.

Gonioscopy This diagnostic test helps determine whether the angle where the iris meets the cornea is open and wide or narrow and closed. During the test, eye drops are used to numb the eye. A hand-held contact lens is gently placed on the eye. This contact lens has a mirror that shows the opthalmologist if the angle between the iris and cornea is closed and blocked (a possible sign of angle-closure or acute glaucoma) or wide and open (a possible sign of open-angle, chronic glaucoma).

Pachymetry is a simple, painless test to measure the thickness of your cornea -- the clear window at the front of the eye. A probe called a pachymeter is gently placed on the front of the eye (the cornea) to measure its thickness. Pachymetry can help your diagnosis, because corneal thickness has the potential to influence eye pressure readings. With this measurement, your doctor can better understand your IOP reading and develop a treatment plan that is right for you. The procedure takes only about a minute to measure both eyes.

\section{LITERATURE REVIEW}

K.Chiranjeevi et al. have proposed an algorithm which detects features in ultrasound images of eye using fundus. They have developed an algorithm to automatically identify clinical features in ultrasound images of the eyes using classification and segmentation techniques. They have used signal processing techniques to locate sclera spur. But the visual quality of the image degrades severly due to speckle noise.To reduce speckle noise they have used multi scale algorithm. They have tested the algorithm on various images of eye and have given comparative studies in tabular form for each image processed. The paper would have been more understable if the clinical parameters used could have been elaborated.Also acronyms used in the paper have not been elaborated, which makes the paper readability a bit tough. The algorithm worked well in $97 \%$ cases where features were correctly extracted in processed image. However, the designed algorithm failed for a few of images, where more noise was present. [4]

Jorg Meier et. Al have focused on a novel automated classification system which is based on image features from fundus photographs. Their research has data driven approach and hence manual assistance is not required. They have discussed on image feature extraction and classification based on statistical evaluation. The paper focuses on the methods they have used for automated classification of the glaucoma affected images of the eyes. They have used principal component analysis for dimension reduction and support vector machine as classifier. The PCA method have 
provided a classification success rate of $81 \%$. They have showed that adequate and disease dependent reprocessing allows an automated appearance based classification comparable to human observers. [5]

D C Hoffman proposed fuzzy model to detect visual field progression. The fuzzy logic model had a sensitivity of $75 \%$ and a specificity of $74 \%$ to detect visual field progression in a series compared with AGIS. A neural network had a sensitivity of $74 \%$ and a specificity of $85 \%$. The agreement between the two was $72.6 \%$ percent agreement, kappa $=.4$ [16].

W. K. Wong et.al have proposed a method to automatically detect CDR(Cup Disc Ratio) using level-set techniques to derive boundaries of the optic cup and disc for fundus images of eye, utilizing initial contours using intensity analysis of the retinal images, without the need for training. This is a key component for ARGALI developed by them for diagnosis of glaucoma risk. 104 retinal images were processed and their CDR was calculated. The proposed method gives a $96 \%$ confidence of obtaining results within a range of \pm 0.2 , which is within the variability for manually graded CDR values. [8] N.Anil Kumar et.al have proposed an algorithm which focuses on automatic detection and determining the exact location of the apex point of the anterior chamber region for efficient angle calculation from the various live ultra sound images. They have developed an algorithm based on active contours to calculate clinical parameters, new region classification and segmentation techniques. The paper focuses on the calculation of Angle Open Distance .They have discussed the various steps of algorithm, but the paper lacks the clinical parameters taken into consideration. The testing images have not been mentioned . [10].

Andrew Turpin et.al paper discusses the data mining methods for checking the progression of glaucoma which is tough as their are no universally accepted definition of glaucomatous progression. Visual field measurements are an essential tool for detecting progression Their paper discusses the decision on classification problem where patients can be classified as stable or progressing.. They have used $1 \mathrm{R}$ and Support vector Machine on visual field test to determine the progression of glaucoma. The standard visual field assessment currently employed in glaucoma management requires the patient to sit facing a half sphere with a white background that encompasses their entire field of vision. The results of their experiments show that both $1 \mathrm{R}$ and support vector machines appear to improve on pointwise univariate regression, a method that is commonly used for glaucoma analysis. [16]

Jiang Liu et.al have proposed an automatic glaucoma diagnosis architecture automatic glaucoma diagnosis through medical imaging informatics (AGLAIA-MII) that combines patient personal data, medical retinal fundus image, and patient's genome information for screening. They had screened 2258 cases from a population study .These cases were attributed with patient personal data, retinal images and quality controlled genome data. Utilizing the multiple kernel learning based classifier, AGLAIA-MII, combined patient personal data, major image features, and important genome single nucleotide polymorphism (SNP) features. They have compared the results obtained from AGLAIA-MII performance with with classifiers using patient personal data, images, and genome SNP separately. AGLAIA-MII was able to achieve an area under curve value of 0.866 , better than $0.551,0.722$ and 0.810 by the individual personal data, image and genome information components, respectively. AGLAIA-MII also demonstrated a substantial improvement over the current glaucoma screening approach based on intraocular pressure. AGLAIA-MII demonstrates for the first time the capability of integrating patients' personal data, medical retinal image and genome information for automatic glaucoma diagnosis and screening in a large dataset from a population study. It paves the way for a holistic approach for automatic objective glaucoma diagnosis and screening. [18]

The authors $\mathrm{Ou}$ Tan and et. Al had 149 patients which were classified into three categories 1) normal 2) perimetric glaucoma (PG), and 3) glaucoma suspect and preperimetric glaucoma. They have used images obtained from the Zeiss Stratus OCT to map the macula over a $6-\mathrm{mm}$ diameter and to scan the circumpapillary nerve fiber layer (cpNFL). These macular OCT images were exported for automatic segmentation using software developed by the authors. The thickness of the macular nerve fiber layer (mNFL), ganglion cell layer (mGCL), inner plexiform layer (mIPL), inner nuclear layer ( $\mathrm{mINL}$ ), outer retinal layer (mORL), and total retinal thickness were measured .Through their research they have found that the $\mathrm{mIRL}$ was the best macular parameter for glaucoma diagnosis and had discriminant power comparable with that of the cpNF. Their findings also suggest that the current Stratus OCT software provides a map of mRetina along with regional averages, but it does not provide thickness measurements of retinal layers. Studies using the Stratus software have shown significant reductions in total macular thickness in glaucomatous eyes compared with normal eyes. [19] A macular segmentation algorithm for OCT was developed by Ishikawa et al. Their results indicate that the macular nerve fiber layer (mNFL) and inner retinal complex (ganglion cell, inner plexiform, and inner nuclear layers) thickness are comparable with cpNFL thickness in discriminating normal eyes from glaucomatous eyes.[20]

\section{CONCLUSION}

Early detection of Glaucoma can prevent early vision loss in the patient. Lowering eye pressure in glaucoma's early stages slows down the progression of the disease and helps save vision Through the extensive literature review carried out it has been observed that though various methods for detection of glaucoma have been carried out there is still a need and scope to develop a Computer Aided System which can not only help diagnose Glaucoma but would also help in checking the progression of the disease so that its growth can be restricted if not prevented. Lot of recent research is being carried for detection of Glaucoma using fundus images, but still detection of progression of Glaucoma in patient remains to be researched. A long-term prospective study is needed to determine the utility of this grading index in assessing glaucoma progression.

Also their is a need to develop a software that segments retinal layers and measures the specific layers involved in glaucoma may improve glaucoma detection.

\section{REFERENCES}

[1] http://www.dnaindia.com/health/report glaucoma-silently-blights-light-in-the-eyes 1519673 
[2] Diagnosis system using data mining approach for Glaucoma (a social threat)- Chauhan, K. Chauhan, P. ; Gulati, R. Technology and Society in Asia (T\&SA), 2012 IEEE Conference pg 1 - 4 e-ISBN 978-1-4673-2068-9

[3] Gardiner SK, Johnson CA, Cioffi GA. Evaluation of the structure-function relationship in glaucoma. Invest Ophthalmol Vis Sci. 2005;46(10):3712-3717.

[4] Automatic Detection of Glaucoma Disease In Eye K.Chiranjeevi et al. / (IJAEST) INTERNATIONAL JOURNAL OF ADVANCED ENGINEERING SCIENCES AND TECHNOLOGIES Vol No. 6, Issue No. 1, 077 - 080

[5] Effects of Preprocessing Eye Fundus Images on Appearance Based Glaucoma Classification Jorg Meier1, Rudiger Bock1, Georg Michelson2, Laszlo G. Nyul1, andJoachim Hornegger

[6] An Efficient Automated System for Detection using Fundus image - K.NARASIMHAN, K.VIJAYAREKH A Journal of Theoretical and Applied Information Technology15th November 2011. Vol. 33 No.1

[7] Relationship between Visual Field Sensitivity and Retinal Nerve Fiber Layer Thickness as Measured by Optical Coherence Tomography -Csilla Ajtony,Zsolt Balla,Szabolcs Somoskeoy,and Balint Kovacs http://www.iovs.org/content/48/1/258.full.pdf+html

[8] LEVEL-SET BASED AUTOMATIC CUP-TO-DISC RATIODETERMINATION USING RETINAL FUNDUS IMAGES IN ARGALID. W. K. Wong1, J. Liu1, J.H. Lim1, X. Jia1, F. Yin1, H. Li1, T. Y. Wong2 30th Annual International IEEE EMBS Conference Vancouver, British Columbia, Canada, August 20-24, 2008

[9] http://www.revoptom.com/content/d/technology/c/19985/

[10] Active Contours Techniques for Automatic Detection of Glaucoma N.Anil Kumar, M. Satya Anuradha, Prakash.SSVD.Vepa, Ravuri Daniel International Journal of Recent Technology and Engineering (IJRTE)ISSN: 2277 3878, Volume-1, Issue-4, October 2012

[11] Integration and fusion of standard automated perimetry and optical coherence tomography data for improved automated glaucoma diagnostics -Dimitrios Bizios*, Anders Heijl and Boel Bengtsson

[12] Regional Relationship Between Retinal Nerve Fiber Layer Thickness and Corresponding Visual Field Sensitivity in Glaucomatous Eyes-Akiyasu Kanamori, MD, PhD; Maiko Naka, MD; Azusa Nagai-Kusuhara, MD, PhD;Yuko Yamada, MD, PhD; Makoto Nakamura, MD, PhD; Akira Negi, MD, PhD

[13] Optical Coherence Tomography Machine Learning Classifiers for Glaucoma Detection: A Preliminary Study - Zvia Burgansky-Eliash, Gadi Wollstein,Tianjiao Chu, Joseph D. Ramsey

[14] http://www.eyeworld.org/article.php?sid=4477

[15] Factors for Glaucoma Progression and the effect of Treatment - M.Cristine Leske ,Anders Heijl ,Mohammed Hussain ,Bo Bengtsson ,Leslie Heymann

[16] Determining Progression in Glaucoma Using Visual Fields Andrew Turpin1, Eibe Frank2, Mark Hall2, Ian H. Witten2, and Chris A.

[17] Johnson1ldentifying Visual Field Progression with Fuzzy Logic Model -DC Hoffman1, DE Gaasterland2 and J Caprioli , Invest Ophthalmol Vis Sci 2002;43: E-Abstract 2169.@ 2002 ARVO

[18] Automatic glaucoma diagnosis through medical imaging informatics Jiang Liu,1 Zhuo Zhang,2,3 Damon Wing Kee Wong,1 Yanwu Xu,1 Fengshou Yin,1 Jun Cheng,1 Ngan Meng Tan,1 Chee Keong Kwoh,3 Dong Xu,3 Yih Chung Tham,4 Tin Aung,5 Tien Yin Wong6 Downloaded from jamia.bmj.com on March 31, 2013

[19] Mapping of Macular Substructures with Optical Coherence Tomography for Glaucoma Diagnosis Ou Tan, PhD, Gisèle Li, MD, Ake Tzu-Hui Lu, PhD, Rohit Varma, MD, MPH, David Huang, MD, PhD, Advanced Imaging for Glaucoma Study Group*Ophthalmology 2008;115:949-956 @ 2008 by the American Academy of Ophthalmology

[20] Ishikawa H, Stein DM, Wollstein G, et al. Macular segmentation with optical coherence tomography. Invest Ophthalmol Vis Sci 2005;46:2012-7

\section{Author' biography with Photo}

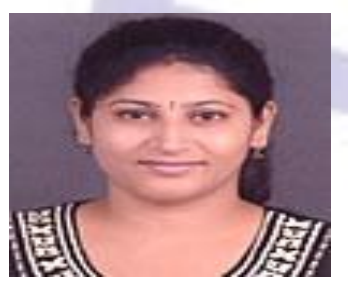

Mrs. Kinjan Chauhan is working as a Assistant Professor in Department of Computer Science, Shree Ramkrishna Inst of Computer Education \& Applied Sciences. She has 9 years of teaching experience. She is pursuing her Doctrate at UKA TARSADIA UNIVERSITY, BARDOLI ,GUJARAT. She has 3 research papers published in peer reviewed International Journals and 6 research paper presentation at various International and National level Conferences.

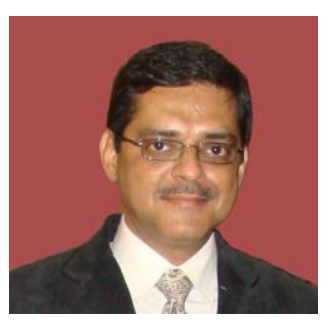

Dr Ravi Gulati is working as an Associate Professor in the Department of Computer Science, Veer Narmad South Gujarat University, Surat. He has teaching experience of around 20 years. He has been associated with M.C.A. and D.C.A. teaching since 1992. $\mathrm{He}$ is also a registered Ph.D. \& M.Phil. supervisor. He has 15 research papers published in peer reviewed International Journals and National Journals. 